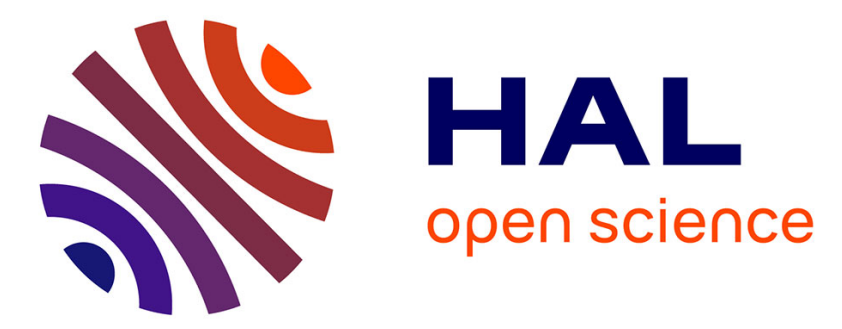

\title{
SOFTWARE FOR RELATIVISTIC ATOMIC THEORY : THE GRASP PROJECT AT OXFORD
}

F. Parpia, I. Grant

\section{To cite this version:}

F. Parpia, I. Grant. SOFTWARE FOR RELATIVISTIC ATOMIC THEORY: THE GRASP PROJECT AT OXFORD. Journal de Physique IV Proceedings, 1991, 01 (C1), pp.C1-33-C1-46. 10.1051/jp4:1991104 . jpa-00249742

HAL Id: jpa-00249742

https://hal.science/jpa-00249742

Submitted on 1 Jan 1991

HAL is a multi-disciplinary open access archive for the deposit and dissemination of scientific research documents, whether they are published or not. The documents may come from teaching and research institutions in France or abroad, or from public or private research centers.
L'archive ouverte pluridisciplinaire HAL, est destinée au dépôt et à la diffusion de documents scientifiques de niveau recherche, publiés ou non, émanant des établissements d'enseignement et de recherche français ou étrangers, des laboratoires publics ou privés. 


\title{
SOFTWARE FOR RELATIVISTIC ATOMIC THEORY : THE GRASP PROJECT AT OXFORD
}

\author{
F.A. PARPIA and I.P. GRANT \\ University of Oxford, Department of Theoretical Chemistry, 5 South \\ Parks Road, GB-Oxford, OXI 3UB, Great-Britain
}

Résumé - GRASP est l'acronyme de General-purpose Relativistic Atomic Structure Program. Ce progiciel est essentiellement un code Dirac-Fock multiconfigurationnel (MCDF) convivial basé sur l'algèbre de Racah et les méthodes de différences finies. Nous décrivons ici brièvement le formalisme MCDF dans le contexte du projet GRASP à Oxford.

\begin{abstract}
GRASP is an acronym for General-purpose Relativistic Atomic Structure Program. This package is essentially a user-friendly multiconfiguration Dirac-Fock (MCDF) code based on the Racah Algebra and finitedifference methods. We describe briefly the MCDF formalism in the context of the GRASP project at Oxford.
\end{abstract}

\section{1 - Introduction}

Relativistic atomic theory is known to provide an economical and reliable approach to the estimation of the static and dynamic properties of highly-charged ions. There are, however, numerous other niches in atomic theory where the relativistic formalism is a felicitous choice: for instance, inner-shell processes in heavy atoms, the calculation of term energies to spectroscopic accuracy, and, quite generally, any situation in which strong configuration mixing is evident.

The needs of physicists investigating processes that occupy the niches best studied with relativistic atomic theory have been addressed to some extent by three software projects at Oxford University. In the following sections of this article we treat only the GRASP structure package. The two othex codes, JJMTRX $\left({ }^{1}\right)$ - a relatjvistic R-matrix

$\left({ }^{1}\right)$ The JJMTRX project is a collaboration between The Queen's University of Belfast and Oxford University. 
suite for electron-atom/ion collision and photoionization calculations - and SWIRLES $\left({ }^{2}\right)$ - a basis-set Dirac-Fock/relativistic many-body perturbation theory structure program will be described elsewhere.

We adopt a naive approach to relativistic atomic structure theory. (Readers wishing to begin from a more rigorous foundation should consult the literature - cf. $/ 1 /$, for instance.) In section 2, we thus build $N$-electron Hamiltonians from one- and two-particle component Hamiltonians. The construction of wavefunctions for $N$-electron atoms from one-electron orbitals is described in broad outline in section 3. A similar method is adopted for the exposition of the multiconfiguration Dirac-Fock (MCDF) method in section 4. The theory is motivated with concrete examples.

\section{2 - Relativistic Hamiltonians}

A Hamiltonian, $\hat{H}$, for the $i=1, \ldots, N$ electrons of an atomic system is a sum of $N$ one-body contributions, $\hat{H}^{(1)}(i)$ and $N(N-1) / 2$ two-body contributions, $\hat{H}^{(2)}(i j)$,

$$
\hat{H}=\sum_{i=1}^{N} \hat{H}^{(1)}(i)+\sum_{\substack{i, j=1 \\ i \neq j}}^{N} \hat{H}^{(2)}(i j)
$$

Symbols representing operators are distinguished by placing carets over them.

The dominant relativistic corrections to atomic structure are ultimately due to modifications to the one-body terms in the Hamiltonian. Current usage therefore classifies the formalism as relativistic when the one-body operators, $\hat{H}^{(1)}(i)$, are based upon the Dirac kinetic energy operator, $\hat{H}^{\mathrm{D}}(i) / 2 /$,

$$
\hat{H}^{\mathrm{D}}(i)=c \overrightarrow{\tilde{\alpha}}(i) \cdot \overrightarrow{\hat{p}}(i)+(\tilde{\beta}(i)-\tilde{1}(i)) c^{2}
$$

Symbols representing matrices are distinguished by placing tildes over them. Here and elsewhere we make use of Hartree atomic units: $e=h / 2 \pi=m_{\mathbf{e}}=1$, whence $c=1 / \alpha$; $e$ is the charge on the proton, $h$ is Planck's constant, $m_{\mathrm{e}}$ is the rest mass of the electron, $c$ is the speed of light in the vacuum and $\alpha \approx 1 / 137$ is the fine-structure constant. In Eq. (2-2), $\overrightarrow{\tilde{\alpha}}(i) \cdot \overrightarrow{\hat{p}}(i)=\tilde{\alpha}_{x}(i) \hat{p}_{x}(i)+\tilde{\alpha}_{y}(i) \hat{p}_{y}(i)+\tilde{\alpha}_{z}(i) \hat{p}_{z}(i)$; the $\tilde{\alpha}$ 's here and the $\tilde{\beta}$ in Eq. (2-2) are Dirac matrices (cf., for instance, $/ 3 /$ ); the $\hat{p}$ 's are the Cartesian components of the momentum operator. When the nucleus is modelled as a spherically-symmetric distribution of charge, the relativistic one-body operator takes the form

$$
\hat{H}^{(1)}(i)=\hat{H}^{\mathrm{D}}(i)+V_{\text {nuc }}(\hat{r}(i)) .
$$

The simplest two-body operator we consider is that arising from the Coulomb interaction,

$$
\hat{H}^{\mathrm{C}}(i j)=\hat{R}^{-1}(i j)
$$

where $\overrightarrow{\hat{R}}(i j)=\overrightarrow{\hat{r}}(i)-\overrightarrow{\hat{r}}(j)$. The resulting Hamiltonian, known as the Dirac-Coulomb Hamiltonian, is denoted $\hat{H}^{\mathrm{DC}}$.

$\left.{ }^{2}\right)$ The SWIRLES project is a collaboration between Oxford University and the Rutherford Appleton Laboratory. 
Quantum electrodynamics (QED) (cf., for instance, /3/) predicts additional twobody interactions. The dominant correction to the Coulomb interaction, due to the exchange of a single transverse photon of energy $\omega$ between a pair of electrons $i$ and $j$, contributes

$$
\begin{aligned}
& \hat{H}^{\text {trans }}(i j)=-\overrightarrow{\tilde{\alpha}}(i) \cdot \overrightarrow{\tilde{\alpha}}(j)\left(\frac{\cos (\omega \hat{R}(i j))}{\hat{R}(i j)}\right) \\
&-(\overrightarrow{\tilde{\alpha}}(i) \cdot \overrightarrow{\tilde{\nabla}}(i))(\overrightarrow{\tilde{\alpha}}(j) \cdot \overrightarrow{\tilde{\nabla}}(j))\left(\frac{\cos (\omega \hat{R}(i j))-1}{\omega^{2} \hat{R}(i j)}\right)
\end{aligned}
$$

to the energy. The $\omega \rightarrow 0$ limit of $\hat{H}^{\text {trans }}$, known as the Breit interaction $/ 4 /$,

$$
\hat{H}^{\mathrm{Breit}}(i j)=-\frac{\overrightarrow{\tilde{\alpha}}(i) \cdot \overrightarrow{\tilde{\alpha}}(j)}{2 \hat{R}(i j)}-\frac{(\overrightarrow{\tilde{\alpha}}(i) \cdot \overrightarrow{\hat{R}}(i j))(\overrightarrow{\tilde{\alpha}}(j) \cdot \overrightarrow{\hat{R}}(i j))}{2 \hat{R}^{3}(i j)}
$$

accounts for the dominant part of the transverse interaction. The Breit interaction is the sum of the Gaunt (or magnetic) interaction $/ 5 /, \hat{H}^{\mathrm{Gaunt}}(i j)=-\overrightarrow{\tilde{\alpha}}(i) \cdot \overrightarrow{\tilde{\alpha}}(j) / \hat{R}(i j)$, and the lowest-order retardation correction to the electrostatic interaction $/ 4 /, H^{\text {ret }}(i j)=$ $\vec{\alpha}_{i} \cdot \vec{\alpha}_{j} / 2 \hat{R}(i j)-[\overrightarrow{\tilde{\alpha}}(i) \cdot \overrightarrow{\hat{R}}(i j)][\overrightarrow{\tilde{\alpha}}(j) \cdot \overrightarrow{\hat{R}}(i j)] / 2 \hat{R}^{3}(i j)$.

Nuclear motion also introduces contributions to the energy. The major effect is a rescaling of the energy of the form

$$
E_{\Gamma} \rightarrow \mu E_{\Gamma}
$$

where $E_{\Gamma}$ is an atomic energy level and $\mu=A /(1+A)$ where $A=M_{\text {nuc }} / m_{\mathrm{e}}$ and $M_{\text {nuc }}$ is the mass of the nucleus.

The theory outlined so far may be inadequate for certain high-precision calculations: the correlated motions of the electrons introduce additional corrections to the nuclear motional energy; the detailed structure of the nucleus has been neglected entirely; we have not considered the influence of the virtual electron-positron pairs which arise from the fluctuation of the vacuum - principally the self-energy and vacuum-polarization corrections. These effects are, however, of higher order than those discussed above and are consistently treated by a perturbation-theory approach to QED (cf., for instance, /1/).

\section{3 - Relativistic Wavefunctions}

Atomic calculations are usually based upon the orbital approximation. In relativistic atomic physics, an orbital, $|n l j m\rangle$, is a simultaneous eigenfunction of the parity operator, $\hat{\pi}$, and the angular momentum operator, $\overrightarrow{\hat{j}}=\overrightarrow{\hat{l}}+\overrightarrow{\hat{s}}$; thus,

$$
\begin{aligned}
\hat{\pi}|n l j m\rangle & =(-1)^{l}|n l j m\rangle \\
\hat{\jmath}^{2}|n l j m\rangle & =j(j+1)|n l j m\rangle \\
\hat{\jmath}_{z}|n l j m\rangle & =m|n l j m\rangle, \quad m=-j, \ldots, j
\end{aligned}
$$


Here $n$ is the principal quantum number. We require that the orbitals form an orthonormal set,

$$
\left\langle n_{a} l_{a} j_{a} m_{a} \mid n_{b} l_{b} j_{b} m_{b}\right\rangle=\delta_{a b} .
$$

The quantum numbers $l, j$ and $m$ provide a complete specification of the symmetry. Each of the $\leq(2 j+1)$ orbitals with the same $j$ but differing $m$ quantum numbers, referred to as a subshell, are assumed to have the same radial form. Using the conventions of $/ 6 /$, an explicit representation is thus

$$
\langle\vec{r} \mid n l j m\rangle=\frac{1}{r}\left(\begin{array}{r}
P_{n l j}(r) \chi_{l j m}(\vec{r} / r) \\
i Q_{n l j}(r) \bar{\chi}_{l j m}(\vec{r} / r)
\end{array}\right) .
$$

Here $P_{n l j}(r)$ and $Q_{n l j}(r)$ are, respectively, the large and small component radial wavefunctions, and the functions $\chi_{l j m}(\vec{r} / r)$ are the spinor spherical harmonics,

$$
\chi_{l j m}(\vec{r} / r)=\sum_{\sigma= \pm \frac{1}{2}}\left\langle l m-\sigma \frac{1}{2} \sigma \mid l \frac{1}{2} j m\right\rangle Y_{l}^{m-\sigma}(\vec{r} / r) \phi^{\sigma}
$$

where $\left\langle l m-\sigma \frac{1}{2} \sigma \mid l \frac{1}{2} j m\right\rangle$ is a Clebsch-Gordan coefficient, $Y_{l}^{m-\sigma}(\vec{r} / r)$ is a spherical harmonic and $\phi^{\sigma}$ is a spinor basis function. For a given value of $j$ the quantum number $l$ can take the values $l=j \pm \frac{1}{2}$. The appropriate barred spinor, $\bar{\chi}_{l j m}$, in Eq. (3-3) is then obtained by choosing $l=j \mp \frac{1}{2}$ in Eq. (3-4).

A configuration state function (CSF), $\left|\gamma J^{P} M\right\rangle$, of an $N$-electron system is formed by linearly combining Slater determinants of order $N$ constructed from the orbitals (3-3) so as to obtain eigenfunctions of the parity operator, $\hat{\Pi}=\hat{\pi}_{1} \cdots \hat{\pi}_{N}$, and total angular momentum operator, $\overrightarrow{\hat{J}}=\overrightarrow{\hat{\jmath}}_{1}+\cdots+\overrightarrow{\hat{\jmath}}_{N} ;$ thus

$$
\begin{aligned}
\hat{I}|\gamma P J M\rangle & =P|\gamma P J M\rangle, \quad P=(-1)^{l_{1}+\cdots+l_{N}}, \\
\hat{J}^{2}|\gamma P J M\rangle & =J(J+1)|\gamma P J M\rangle, \\
\hat{J}_{z}|\gamma P J M\rangle & =M|\gamma P J M\rangle, \quad M=-J, \ldots, J .
\end{aligned}
$$

Any additional information required to uniquely specify the construction of the CSF is represented by the symbol $\gamma$. We require orthonormality of the CSFs, $\left\langle\gamma_{r} J_{r}^{P_{r}} M_{r} \mid \gamma_{s} J_{s}^{P_{s}} M_{s}\right\rangle=\delta_{r s}$.

An (approximate) atomic state function (ASF), $\left|\Gamma J^{P} M\right\rangle$, is a linear combination of CSFs with the given eigenvalues $J, P$, and $M$. Because the transformation matrix elements, $c_{r} \Gamma=\left\langle\gamma_{r} J^{P} M \mid \Gamma J^{P} M\right\rangle$, are independent of the quantum number $M$, the entire set of $(2 \mathrm{~J}+1)$ transformation equations may be written

$$
\left|\Gamma J^{P}\right\rangle=\sum_{r=1}^{n_{J} P} c_{r \Gamma}\left|\gamma_{r} J^{P}\right\rangle
$$

$n_{J^{P}}$ is then the number of levels with quantum numbers $J$ and $P$. The mixing coefficients, $c_{r} \Gamma$, may be combined in a column vector, $\tilde{c}_{\Gamma} \equiv\left\{c_{r \Gamma}, r=1, \ldots, n_{J^{P}}\right\}$, the representation of the ASF $\left|\Gamma J^{P} M\right\rangle$ with respect to the CSFs $\left|\gamma_{r} J^{P} M\right\rangle, r=1, \ldots, n_{J^{P}}$. This procedure effectively partitions the space of CSFs into subspaces characterised by states sharing the eigenvalues $J, P$ and $M$. The ASFs will be chosen to be orthonormal, so that

$$
\left(\tilde{c}_{\Gamma_{i}}\right)^{\dagger} \tilde{c}_{\Gamma_{j}}=\delta_{i j}
$$


the dagger denotes the Hermitian conjugate.

\section{4 - GRASP}

Approximate eigenenergies (or first-order perturbative corrections to approximate eigenenergies) of free atoms are then obtained as diagonal expectation values of the approximate Hamiltonians (or perturbation Hamiltonians) with respect to ASFs. For instance,

$$
E_{\Gamma}^{\mathrm{DC}} \equiv\left\langle\Gamma J^{P} M\left|\hat{H}^{\mathrm{DC}}\right| \Gamma J^{P} M\right\rangle
$$

These energies are independent of the eigenvalue $M$ by construction, and are therefore $(2 J+1)$-fold degenerate.

The Dirac-Coulomb Hamiltonian matrix, $\tilde{H}^{\mathrm{DC}}$, with elements

$$
H_{r s}^{\mathrm{DC}}=\left\langle\gamma_{r} J_{r}^{P_{r}} M_{r}\left|\hat{H}^{\mathrm{DC}}\right| \gamma_{s} J_{s}^{P_{s}} M_{s}\right\rangle \delta_{J_{r} J_{s}} \delta_{P_{r} P_{s}} \delta_{M_{r} M_{s}}
$$

plays a central role in the GRASP implementation of the multiconfiguration Dirac-Fock (MCDF) theory. The matrix elements $H_{r s}^{\mathrm{DC}}$ are also independent of $M$ with a degeneracy of $(2 J+1)$. They may be written as sums of products of angular coefficients and radial integrals,

$$
H_{r s}^{\mathrm{DC}}=\sum_{i=1}^{N_{r s}^{(T)}} T_{r s}\left(a_{i} b_{i}\right) I\left(a_{i} b_{i}\right)+\sum_{i=1}^{N_{r s}^{(V)}} V_{r s}^{\left(k_{i}\right)}\left(a_{i} b_{i} c_{i} d_{i}\right) R^{\left(k_{i}\right)}\left(a_{i} b_{i} c_{i} d_{i}\right)
$$

The one-body contributions (2-3) give rise to the $T_{r s}(a b)$ angular coefficients and the $I(a b)$ radial integrals,

$$
\begin{aligned}
I(a b)=\delta_{l_{a} l_{b}} \delta_{j_{a} j_{b}} \int_{0}^{\infty} d r\left[c\left(Q_{n_{a} l_{a} j_{a}}(r) P_{n_{b} l_{b} j_{b}}^{\prime}(r)-P_{n_{a} l_{a} j_{a}}(r) Q_{n_{b} l_{b} j_{b}}^{\prime}(r)\right)\right. \\
\\
-2 c^{2} Q_{n_{a} l_{a} j_{a}}(r) Q_{n_{b} l_{b} j_{b}}(r) \\
\quad+\frac{c \kappa_{a}}{r}\left(P_{n_{a} l_{a} j_{a}}(r) Q_{n_{b} l_{b} j_{b}}(r)+Q_{n_{a} l_{a} j_{a}}(r) P_{n_{b} l_{b} j_{b}}(r)\right) \\
+ \\
\left.+V_{\mathrm{nuc}}(r)\left(P_{n_{a} l_{a} j_{a}}(r) P_{n_{b} l_{b} j_{b}}(r)+Q_{n_{a} l_{a} j_{a}}(r) Q_{n_{b} l_{b} j_{b}}(r)\right)\right]
\end{aligned}
$$

where $f^{\prime} \equiv d f / d r$ and $\kappa=-(2 j+1)(j-l)$; the interelectronic interactions (2-4) yield the $V_{r s}^{(k)}(a b c d)$ angular coefficients and the relativistic Slater integrals, $R^{(k)}(a b c d)$,

$$
R^{(k)}(a b c d)=\int_{0}^{\infty} d r\left[\left(P_{n_{a} l_{a} j_{a}}(r) P_{n_{c} l_{c} j_{c}}(r)+Q_{n_{a} l_{a} j_{a}}(r) Q_{n_{c} l_{c} j_{c}}(r)\right) \frac{1}{r} Y^{(k)}(b d ; r)\right]
$$

the relativistic Hartree $Y$-functions are defined by the equation

$$
Y^{(k)}(a b ; r)=r \int_{0}^{\infty} d s \frac{r_{<}^{k}}{r_{>}^{k+1}}\left(P_{n_{a} l_{a} j_{a}}(s) P_{n_{b} l_{b} j_{b}}(s)+Q_{n_{a} l_{a} j_{a}}(s) Q_{n_{b} l_{b} j_{b}}(s)\right)
$$

Here $r_{>}\left(r_{<}\right)$denotes the greater (lesser) of $r$ and $s$. 
A prerequisite for the development of a Racah-algebra based program to compute the angular coefficients $T_{r s}(a b)$ and $V_{r s}^{(k)}(a b c d)$ is a code to determine $j j$-coupled coefficients of fractional parentage. Such a program, CFPJJ /7/, was made available through the Computer Physics Communications Program Library in 1972.

A second package, MCP /8/, to compute the $V_{r s}^{(k)}(a b c d)$ coefficients, followed shortly thereafter, in 1973. This code was revised and reissued as MCP75/9/ in 1976. Either MCP package accepts a list of CSF specifications $\left(\gamma_{r} J_{r}^{P_{r}}\right)$ (specifying the value of $M$ is unnecessary - cf. Eq. (4-2)) and computes the value, $V_{r s}^{\left(k_{i}\right)}\left(a_{i} b_{i} c_{i} d_{i}\right)$, and all indices, $k_{i}$, $a_{i}, b_{i}, c_{i}, d_{i}$, of each coefficient for each pair $\gamma_{r}$ and $\gamma_{s}$ that interact: i.e., have nonzero matrix elements $H_{r s}$. The total number of coefficients for such a pair is denoted $N_{r s}^{(V)}$ (cf. Eq. (4-3)). (The MCP package used in the recent versions of the Oxford MCDF packages are modified to compute only those coefficients that cannot be expressed in very simple analytical forms.) The user-friendly input interface as implemented in the module CSFIN in MCP is the precursor of the current GRASP interface routine CARDIN.

A major rewrite /10/ of the RDMEJJ package of Chang/11/, now called MCT, was released in 1978 . This code computes angular coefficients, $T_{r s}^{(k \pi)}(a b)$, required to calculate matrix elements of a general one-particle tensor operator of rank $k$ and parity $\pi$,

$$
\hat{F}^{(k \pi)}=\sum_{i=1}^{N} \hat{f}_{q}^{(k \pi)}(i)
$$

in a CSF basis,

$$
\begin{aligned}
\left\langle\gamma_{r} J_{r}^{P_{r}} M_{r}\left|\hat{F}^{(k \pi)}\right| \gamma_{s} J_{s}^{P_{s}} M_{s}\right\rangle= & (-1)^{J_{r}-M_{r}}\left(\begin{array}{ccc}
J_{r} & k & J_{s} \\
M_{r} & q & -M_{s}
\end{array}\right) \\
& \times \sum_{i=1}^{N_{r s}^{\left(T^{(k \pi)}\right)}} T_{r s}^{(k \pi)}\left(a_{i} b_{i}\right)\left(n_{a_{i}} l_{a_{i}} j_{a_{i}}\left|\hat{f}^{(k \pi)}(r)\right| n_{b_{i}} l_{b_{i}} j_{b_{i}}\right) .
\end{aligned}
$$

The coefficients $T_{r s}(a b)$ in Eq. (4-3) are those appropriate to scalar operators $(k=0$, $\pi=+1)$.

The requisite triad of angular packages for MCDF computations was completed in 1980 with a generalization (published as part of MCBP/BENA /12/) of the original MCBP package $/ 13 /$ to finite photon frequencies. The present version of MCBP computes the angular coefficients $V_{r s}^{(k \tau)}(a b c d)$ for the evaluation of the matrix elements of the transverse interaction (2-5),

$$
\begin{aligned}
H_{r s}^{\mathrm{transv}} & =\left\langle\gamma_{r} J_{r}^{P_{r}} M_{r}\left|\hat{H}^{\mathrm{transv}}\right| \gamma_{s} J_{s}^{P_{s}} M_{s}\right\rangle \delta_{J_{r} J_{s}} \delta_{P_{r} P_{s}} \delta_{M_{r} M_{s}} \\
& =\sum_{i=1}^{N_{r s}^{\mathrm{transv}}} V_{r s}^{\left(k_{i} \tau_{i}\right)}\left(a_{i} b_{i} c_{i} d_{i}\right) S_{r s}^{\left(k_{i} \tau_{i}\right)}\left(a_{i} b_{i} c_{i} d_{i}\right)
\end{aligned}
$$

The $S_{r s}^{\left(k_{i} r_{i}\right)}\left(a_{i} b_{i} c_{i} d_{i}\right)$ are radial integrals /14/ that are appreciably more complex than the Slater integrals given by Eq. (4-5).

With the appropriate generalization of a Dirac-Fock $\left(\mathrm{DF}\left({ }^{3}\right)\right)$ self-cousistent field (SCF) code due to D.F. Mayers, it was possible to construct the MCDF+MCBP/BENA /15,12/ pair of packages, which were published in 1980 .

$\left({ }^{3}\right)$ The Dirac-Fock model is obtained when a single Slater determinant can be used to model the atomic state. 
GRASP /16/, published in 1989, is a major rewrite of the MCDF+MCBP/BENA $/ 15,12 /$ package. The portability and currency of the program have been greatly enhanced by recoding from FORTRAN IV to approach the FORTRAN 77 standard. A preprocessor forms an important part of the package: the source code is issued in raw form which must be preprocessed to FORTRAN source code - the preprocessor performs basic editing operations using data from a user-created symbol file to select machine- and installationdependent code and to set array dimensions; the problem to be solved is thus limited only by the stability of the SCF solver and the available storage. Both the (rewritten) MCDF /15/ and MCBP/BENA /12/ modules as well as the TRANSFORM /17/ and OSCL packages have been integrated into the stand-alone GRASP code; using the facilities available in TRANSFORM /17/ the user can perform recoupling from the standard $j j$ coupling of GRASP to nonstandard $j j-, L S$ - or $j l$-coupled eigenvector bases; the OSCL package computes radiative transition data with retardation to all orders. 'The increased capability has necessitated modifications to the interface which has also been rendered more flexible and easy to use. Much effort has been expended to improve efficiency: the Jucys-graph based NJGRAF /18/ package replaces the algebra-based NJSYM /19/ for the computation of angular recoupling coefficients; the SCF algorithm is faster and more stable; the $V_{r s}^{(k)}(a b c d)$ and $T_{r s}^{(k \pi)}(a b)$ coefficients are sorted into an integral-based, rather than a CSF-based list - for instance, it is more efficient to compute the radial integrals in (4-3) only once and then accumulate their contributions to each matrix element $H_{r s}^{\text {DC }}$ rather than to compute all required integrals each time a matrix element is to be computed; radial integrals can be stored - this obviates the need to recompute a part of them if some of the orbitals remain unchanged between calculations. Variations on the MCDF method the freezing of certain orbitals and the Slater exchange approximation, for instance - are available. By the use of the integral scaling facility, GRASP eigenvalues may be made to match experimental data. The accuracy and reliability of the results have been improved - all errors known prior to publishing have been eliminated and more accurate numerical methods are used. The program is internally documented to some extent with plentiful comment cards. A User's Manual provides help in the set-up and use of the package, complete specification of the input and some information about the program's structure and operation.

GRASP $^{2}$ (PARPIA, F.A., GRANT, I.P., DYALL, K.G., and FISCHER, C.F., in preparation) is a major rewrite of GRASP. It is based on a completely new SCF algorithm adapted from procedures developed and extensively used by Fischer (cf., for instance, /20/) for the nonrelativistic multiconfiguration Hartree-Fock method. Testing has revealed that great gains have been made, both in stability and in efficiency, when GRASP ${ }^{2}$ is compared with its predecessors. Full FORTRAN 77 compatibility has been attained. Some minor errors carried over from GRASP have been eliminated. The relativistic local-density approximation for exchange and correlation effects (cf. $/ 21 /$, for instance) is available as an option. The User's Manual that accompanies the program is now a complete reference document: besides a set of tutorials on the set-up and use of the package and a detailed description of the input Command/Data File structure, the underlying physical and numerical formalism, the flow structure and each subroutine of the code are explained. The GRASP $^{2}$ package is in the final stages of testing and should be available before the end of 1990.

GRASP $^{3}$, now in the early stages of development, will extend the capabilities of GRASP $^{2}$ to the calculation of dynamic phenomena (photoionization, electron-atom/ion 
scattering, etc.) involving a single continuum electron. We expect that this program will be released by the end of 1992 .

We motivate the principal classes of MCDF procedures as implemented in GRASP ${ }^{2}$ with two examples. Consider the estimation of the energies of the seven lowest-lying levels of heliumlike Argon, $\mathrm{Ar}^{16+}$,

$$
\begin{aligned}
& \left|\left(00000000.00 \mathrm{~cm}^{-1}\right) 10^{+}\right\rangle \stackrel{c \rightarrow \infty}{\rightarrow}\left|1 \mathrm{~s}^{2}{ }^{1} \mathrm{~S}_{0}^{\mathrm{e}}\right\rangle, \\
& \left|\left(25036594.11 \mathrm{~cm}^{-1}\right) 21^{+}\right\rangle \stackrel{c \rightarrow \infty}{\longrightarrow}\left|1 \mathrm{~s} 2 \mathrm{~s}^{3} \mathrm{~S}_{1}^{\mathrm{e}}\right\rangle, \\
& \left|\left(25187779.82 \mathrm{~cm}^{-1}\right) 20^{-}\right\rangle \stackrel{c \rightarrow \infty}{\longrightarrow}\left|1 \mathrm{~s} 2 \mathrm{p}^{3} \mathrm{P}_{0}^{o}\right\rangle, \\
& \left|\left(25192958.84 \mathrm{~cm}^{-1}\right) 21^{-}\right\rangle \stackrel{c \rightarrow \infty}{\longrightarrow}\left|1 \mathrm{~s} 2 \mathrm{p}^{3} \mathrm{P}_{1}^{o}\right\rangle, \\
& \left|\left(25200959.64 \mathrm{~cm}^{-1}\right) 20^{+}\right\rangle \stackrel{c \rightarrow \infty}{\longrightarrow}\left|1 \mathrm{~s} 2 \mathrm{~s}^{1} \mathrm{~S}_{0}^{\mathrm{e}}\right\rangle, \\
& \left|\left(25215171.18 \mathrm{~cm}^{-1}\right) 22^{-}\right\rangle \stackrel{c \rightarrow \infty}{\longrightarrow}\left|1 \mathrm{~s} 2 \mathrm{p}^{3} \mathrm{P}_{2}^{o}\right\rangle, \\
& \left|\left(25322387.43 \mathrm{~cm}^{-1}\right) 21^{-}\right\rangle \stackrel{c \rightarrow \infty}{\longrightarrow}\left|1 \mathrm{~s} 2 \mathrm{p}^{1} \mathrm{P}_{1}^{o}\right\rangle,
\end{aligned}
$$

where the excitation energy with respect to the ground state (computed from the ionization energies in $\mathrm{cm}^{-1}$ as given by Drake /22/) appears in parentheses on the left-hand side, and the designation of the state in the nonrelativistic limit is given on the right-hand side, of each line. For this problem, it is well known that the most important CSFs are, respectively,

$$
\begin{aligned}
& \left|10^{+}\right\rangle \approx\left|1 \mathrm{~s}_{1 / 2}^{2} 0^{+}\right\rangle \\
& \left|21^{+}\right\rangle \approx\left|1 \mathrm{~s}_{1 / 2} 2 \mathrm{~s}_{1 / 2} 1^{+}\right\rangle \\
& \left|20^{-}\right\rangle \approx\left|1 \mathrm{~s}_{1 / 2} 2 \mathrm{p}_{1 / 2} 0^{-}\right\rangle \\
& \left|21^{-}\right\rangle \approx a\left|1 \mathrm{~s}_{1 / 2} 2 \mathrm{p}_{1 / 2} 1^{-}\right\rangle+b\left|1 \mathrm{~s}_{1 / 2} 2 \mathrm{p}_{3 / 2} 1^{-}\right\rangle \\
& \left|20^{+}\right\rangle \approx\left|1 \mathrm{~s}_{1 / 2} 2 \mathrm{~s}_{1 / 2} 0^{+}\right\rangle \\
& \left|22^{-}\right\rangle \approx\left|1 \mathrm{~s}_{1 / 2} 2 \mathrm{p}_{3 / 2} 2^{-}\right\rangle \\
& \left|21^{-}\right\rangle \approx c\left|1 \mathrm{~s}_{1 / 2} 2 \mathrm{p}_{3 / 2} 1^{-}\right\rangle+d\left|1 \mathrm{~s}_{1 / 2} 2 \mathrm{p}_{1 / 2} 1^{-}\right\rangle
\end{aligned}
$$

(The quantum number $M$ has been suppressed on both sides of each approximate equality.) An array of $7^{2}=49$ elements is needed to store all unique values of $H_{r s}^{\mathrm{DC}}$,

$$
\tilde{H}^{\mathrm{DC}}=\left(\begin{array}{ccccccc}
H_{11}^{\mathrm{DC}} & 0 & 0 & 0 & H_{15}^{\mathrm{DC}} & 0 & 0 \\
0 & H_{22}^{\mathrm{DC}} & 0 & 0 & 0 & 0 & 0 \\
0 & 0 & H_{33}^{\mathrm{DC}} & 0 & 0 & 0 & 0 \\
0 & 0 & 0 & H_{44}^{\mathrm{DC}} & 0 & 0 & H_{47}^{\mathrm{DC}} \\
H_{51}^{\mathrm{DC}} & 0 & 0 & 0 & H_{55}^{\mathrm{DC}} & 0 & 0 \\
0 & 0 & 0 & 0 & 0 & H_{66}^{\mathrm{DC}} & 0 \\
0 & 0 & 0 & H_{74}^{\mathrm{DC}} & 0 & 0 & H_{77}^{\mathrm{DC}}
\end{array}\right)
$$

where the order of the CSFs is as they appear in the first term on the right in each line of Eq. (4-11). This matrix is actually symmetric (by virtue of the phase convention adopted in the GRASP project; the matrix would be Hermitian otherwise - cf. Eq. (4-4)). It is therefore necessary to compute only 28 of the 49 elements. Those set to zero must take this value because of the selection rules on parity and angular momentum (explicitly shown in $\mathrm{Eq} \cdot(4-2))$. 
TIME 100

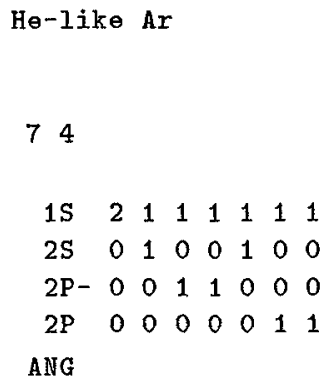

OSCL
Guess that $100 \mathrm{~s}$ of CPU time is required - this strongly depends on the computer employed.

This string is written as an identifier in all GRASP ${ }^{2}$ files (including the print output). The title card also delimits the beginning of a case.

The basic dimensions of the case: $r=1, \ldots, n_{\mathrm{c}}=7$ and $a=1, \ldots, n_{\mathrm{w}}=4$.

Each column here gives the occupation $q_{r}(a)$ of electrons in the four subshells $n_{a} l_{a} j_{a}$ in a given $\gamma_{r} J_{r}^{P_{r}} ; 2 \mathrm{P}$ - represents the $2 \mathrm{p}_{1 / 2}$ subshell, $2 \mathrm{P}$, the $2 \mathrm{p}_{3 / 2}$ subshell.

Any additional information required to complete the specification of the $\gamma_{r} J_{r}^{P_{r}}$ follows this card.

Very little additional information is required for the twoelectron problem - none if the subshell is full, as it is for $r=1$, and only the total angular momentum $J_{r}$ obtained by coupling the angular momenta $j_{a}$ otherwise. The first number in each row is $r$, the second is $J_{r}$.

Compute the $V_{r s}^{(k)}(a b c d)$ and $T_{r s}(a b)$ coefficients; write the CSF-based list on unit 7, the integral-based list on unit 8 . Generate the radial functions using the $\mathrm{AL}$ approximation; read the integral-based list of $V_{r s}^{(k)}(a b c d)$ and $T_{r s}(a b)$ coefficients on unit 8; there is no MCDF Dump File from which the SCF procedure can be restarted - hence the 0 ; write the MCDF Dump File on unit 9; the atomic number of Argon is 18; the atomic weight, 39.948 amu, is used internally to estimate both the nuclear radius and the reduced mass of the electron.

Compute the $V_{r s}^{(k)}(a b c d)$ coefficients and write them out on unit 12. No sorting is done.

Compute the higher-order corrections: diagonalise $\hat{H}^{\text {trans }}$ and estimate the self-energy and vacuum-polarization contributions.

Read the integral-based list of $V_{r s}^{(k)}(a b c d)$ and $T_{r} s(a b)$ coefficients on unit 8, the MCDF Dump File on unit 9 and the $V_{r s}^{(k \tau)}(a b c d)$ coefficients on unit 12 .

Compute the $T_{r s}^{(k \pi)}(a b)$ coefficients for $k=1$, and odd parity; write the CSF-based list on unit 11 and the integral-based list on unit 12 ; there is no restart file from which any CSF-based list can be read: hence the 0 .

Compute electromagnetic transition data: oscillator strengths, Einstein $A$ and $B$ coefficients, etc.

Read the MCDF Dump File on unit 9 and the $T_{r s}^{(k \pi)}(a b c d)$ coefficients on unit 12 .

This card delimits the end of a case.

Figure 1. A typical GRASP ${ }^{2}$ Command/Data File. Each card is briefly explained with the comments that appear to the right of it. A detailed description of the overall structure and the structure of each individual card of the Command/Data File may be found in the User's Manual that is issued with the program. 
We make use of the member with $M=0$ in Eq. (4-1) - this value of $M$ is universal to all states in systems with an even number of electrons (we would similarly use $M= \pm \frac{1}{2}$ for cases with an odd number of electrons). The energies of all seven atomic levels are then given by equations of the form (cf. Eq. (4-11))

$$
E_{\Gamma}^{\mathrm{DC}}=\left\langle\Gamma J^{P} 0\left|\hat{H}^{\mathrm{DC}}\right| \Gamma J^{P} 0\right\rangle=\left(\tilde{c}_{\Gamma}^{\mathrm{DC}}\right)^{\dagger} \tilde{H}^{\mathrm{DC}} \tilde{c}_{\Gamma}^{\mathrm{DC}}
$$

The optimal values of the mixing coefficient vectors, $\tilde{c}$ are obtained by requiring $E_{\Gamma}^{\mathrm{DC}}$ to be stationary with respect to variations in the mixing coefficients subject to Eq. (3-7). This leads to an eigenvalue problem,

$$
\left(\tilde{H}^{\mathrm{DC}}-E_{\Gamma}^{\mathrm{DC}} \tilde{1}\right) \tilde{c}_{\Gamma}^{\mathrm{DC}}=0 .
$$

Here $\tilde{1}$ is the $n_{\mathrm{c}} \times n_{\mathrm{c}}$ unit matrix, where $n_{\mathrm{c}}=\sum_{J^{P}} n_{J^{P}}$ and we have appropriately extended the mixing coefficient vectors with elements 0 for components in orthogonal subspaces.

The eigenvalue problem given by Eq. (4-14) above can he solved once the matrix $\tilde{H}^{\mathrm{DC}}$ has been constructed. We have seen that this requires knowledge of the angular coefficients $V_{r s}^{(k)}(a b c d)$ and $T_{r s}(a b)$ and the orbital radial functions $P_{n l j}(r), Q_{n l j}(r)$, so that the diagonalization (4-14) is the last step, and the determination of the Hamiltonian matrix (4-12) the penultimate one, in any computational procedure based on the formalism outlined so far.

The first computational step is performed by MCP (which in turn calls the relevant subroutines in MCT) to determine the angular coefficients $V_{r s}^{(k)}(a b c d)$ and $T_{r s}(a b)$; these coefficients are written to a disk file: it is economical to break up an atomic structure problem into its radial and angular parts - one can thus avoid recomputing the angular coefficients for calculations along an isoelectronic sequence, for instance.

The problem of the selection of the functions $P_{n l j}(r), Q_{n l j}(r)$ now arises. These are determined as the numerical solutions of coupled pairs of radial Dirac equations of the form

$$
\begin{gathered}
\left(\frac{d}{d r}+\frac{\kappa_{a}}{r}\right) P_{n_{a} l_{a} j_{a}}(r)-\left(2 c-\frac{\epsilon_{a a}}{c}+\frac{\mathcal{Y}_{a}(r)}{c r}\right) Q_{n_{a} l_{a} j_{a}}(r)=-\frac{\mathcal{X}_{a}^{(P)}(r)}{r} \\
\left(\frac{d}{d r}-\frac{\kappa_{a}}{r}\right) Q_{n_{a} l_{a} j_{a}}(r)+\left(-\frac{\epsilon_{a a}}{c}+\frac{\mathcal{Y}_{a}(r)}{c r}\right) P_{n_{a} l_{a} j_{a}}(r)=-\frac{\mathcal{X}_{a}^{(Q)}(r)}{r}
\end{gathered}
$$

with $\epsilon_{a a}>0$ subject to the boundary conditions

$$
P_{n_{a} l_{a} j_{a}}(r), Q_{n_{a} l_{a} j_{a}}(r) \stackrel{r \rightarrow 0}{\longrightarrow} 0, \quad P_{n_{a} l_{a} j_{a}}(r), Q_{n_{a} l_{a} j_{a}}(r) \stackrel{r \rightarrow \infty}{\longrightarrow} 0
$$

the orthonormalization condition which follows from Eq. (3-2),

$$
\begin{aligned}
& \mathcal{N}(a b)= \begin{cases}0 & \text { when } a \neq b \text { but } l_{a}=l_{b} \text { and } j_{a}=j_{b} \\
1 & \text { when } a=b\end{cases} \\
& \mathcal{N}(a b) \equiv \int_{0}^{\infty} d r\left(P_{n_{a} l_{a} j_{a}}(r) P_{n_{b} l_{b} j_{b}}(r)+Q_{n_{a} l_{a} j_{a}}(r) Q_{n_{b} l_{b} j_{b}}(r)\right)
\end{aligned}
$$

and that the sign of the first oscillation of $P_{n_{a} l_{\alpha} j_{a}}(r)$ that exceeds a certain amplitude be positive and that the total number of such oscillations be $n_{a}-l_{a}-1$ (cf. $/ 20 /$, for instance). 
The asymptotic forms of, and the relation between, the large- and small-component functions near the origin are dependent upon the detailed behaviour of the functions $\mathcal{Y}_{a}(r)$, $\mathcal{X}_{a}^{(P)}(r)$ and $\mathcal{X}_{a}^{(Q)}(r)$ in this neighbourhood.

The latter functions are given by the expressions

$$
\begin{aligned}
& \mathcal{Y}_{a}(r)=-r V_{\text {nuc }}(r)-\sum_{i=1}^{N_{a}^{(y)}} y_{a}^{\left(k_{i}\right)}\left(b_{i} c_{i}\right) Y^{\left(k_{i}\right)}\left(b_{i} c_{i} ; r\right) \\
& \mathcal{X}_{a}^{(P)}(r)=\frac{1}{c} \sum_{i=1}^{N_{a}^{(x)}} x_{a}^{\left(k_{i}\right)}\left(b_{i} c_{i} d_{i}\right) Y^{\left(k_{i}\right)}\left(b_{i} c_{i} ; r\right) Q_{n_{d_{i}} l_{d_{i}} j_{d_{i}}}(r)+\frac{r}{c \bar{q}(a)} \sum_{i=1}^{N_{a}^{(\epsilon)}} \epsilon_{a b_{i}} Q_{n_{b_{i}} l_{b_{i} j_{b_{i}}}}(r) \\
& +r \sum_{i=1}^{N_{a}^{(z)}} z_{a}\left(b_{i}\right)\left[\left(\frac{d}{d r}+\frac{\kappa_{b_{i}}}{r}\right) P_{n_{b_{i}} l_{b_{i}} j_{b_{i}}}(r)-\left(2 c-\frac{V_{\mathrm{nuc}}(r)}{c}\right) Q_{\left.n_{b_{i} b_{b_{i}} j_{b_{i}}}(r)\right]},\right. \\
& \mathcal{X}_{a}^{(Q)}(r)=-\frac{1}{c} \sum_{i=1}^{N_{a}^{(x)}} x_{a}^{\left(k_{i}\right)}\left(b_{i} c_{i} d_{i}\right) Y^{\left(k_{i}\right)}\left(b_{i} c_{i} ; r\right) P_{n_{d_{i}} l_{d_{i}} j_{d_{i}}}(r)-\frac{r}{c \bar{q}(a)} \sum_{i=1}^{N_{a}^{(\epsilon)}} \epsilon_{a b_{i}} P_{n_{b_{i}} l_{b_{i}} j_{b_{i}}}(r) \\
& +r \sum_{i=1}^{N_{a}^{(z)}} z_{a}\left(b_{i}\right)\left[\left(\frac{d}{d r}-\frac{\kappa_{b_{i}}}{r}\right) Q_{n_{b_{i}} l_{b_{i}} j_{b_{i}}}(r)-\left(\frac{V_{\mathrm{nuc}}(r)}{c}\right) P_{n_{b_{i}} l_{b_{i}} j_{b_{i}}}(r)\right] .
\end{aligned}
$$

It is in the determination of the coefficients $x_{a}^{(k)}(b c d), y_{a}^{(k)}(b c)$ and $z_{a}(b)$ that the MCDF procedure is applied. We use a variational functional defined by the weighted energies

$$
\begin{aligned}
W^{\mathrm{DC}} & =\sum_{i=1}^{n_{\mathbf{L}}} w_{i} E_{\Gamma_{i}}^{\mathrm{DC}} / \sum_{i=1}^{n_{\mathrm{L}}} w_{i} \\
& =\sum_{i=1}^{n_{\mathbf{L}}} w_{i} \sum_{r, s=1}^{n_{\mathrm{c}}} c_{r \Gamma_{i}} c_{s \Gamma_{i}} H_{r s} / \sum_{i=1}^{n_{\mathrm{L}}} w_{i} \equiv \sum_{r, s=1}^{n_{\mathrm{c}}} d_{r s} H_{r s}
\end{aligned}
$$

variation with respect to one pair of radial functions, $P_{n_{a} l_{a} j_{a}}(r)$ and $Q_{n_{a} l_{a} j_{a}}(r)$, subject to the boundary conditions (4-16) produces one pair of equations (4-15), and determines all coefficients, $x_{a}^{(k)}(b c d), y_{a}^{(k)}(b c)$ and $z_{a}(b)$, in (4-18). Lagrange multipliers, $\epsilon_{a b}$, are introduced to enforce Eqs. (4-17). The generalised occupation number of an orbital is $\bar{q}(a)$, given by $\sum_{r=1}^{n_{\mathrm{c}}} d_{r r} q_{r}(a)$; here $q_{r}(a)$ is the number of electrons in subshell $\left(n_{a} l_{a} j_{a}\right)$ in CSF set $\left|\gamma_{r} J_{r}^{P_{r}}\right\rangle$.

Equations (4-14) and (4-15) are simultaneously solved using an SCF procedure:

1. The coefficients $d_{r s}$ in (4-19) are calculated from a set of mixing coefficient vectors $\tilde{c}_{\Gamma i}^{\text {est }}, i=1, \ldots, n_{\mathrm{L}}$.

2. The functions $\mathcal{Y}_{a}(r), \mathcal{X}_{a}^{(P)}(r)$ and $\mathcal{X}_{a}^{(Q)}(r)$ in Eq. (4-18) can then be calculated from a set of radial functions $P_{n_{a} l_{a} j_{a}}^{\text {est }}(r), Q_{n_{a} l_{a} j_{a}}^{\text {est }}(r), a=1, \ldots, n_{\mathrm{w}}$.

3. Equations (4-15) are solved using this potential to obtain a new set of radial functions $P_{n_{a} l_{a} j_{a}}^{\text {new }}(r), Q_{n_{a} l_{a} j_{a}}^{\text {new }}(r), a=1, \ldots, n_{\mathrm{w}}$.

4. An improved estimated set of radial functions is obtained from the transformation $\left[P_{n_{a} l_{a} j_{a}}^{\text {est }}(r), Q_{n_{a} l_{a} j_{a}}^{\text {est }}(r)\right] \rightarrow\left(1-\eta_{a}\right)\left[P_{n_{a} l_{a} j_{a}}^{\text {new }}(r), Q_{n_{a} l_{a} j_{a}}^{\text {new }}(r)\right]+\eta_{a}\left[P_{n_{a} l_{a} j_{a}}^{\text {est }}(r), Q_{n_{a} l_{a} j_{a}}^{\text {est }}(r)\right]$, where $0 \leq \eta_{a}<1$ are damping or accelerating factors. If the improved estimated set of orbitals agrees to within a specified tolerance with the original estimated set, convergence has been achieved. If not, steps 5,6 and $1-4$ are executed again. 
5. Equation (4-14) is solved using the Hamiltonian obtained with the improved estimated radial functions to calculate a new estimated set of mixing-coefficient vectors $\tilde{c}_{\Gamma_{i}}^{\text {new }}, i=1, \ldots, n_{\mathrm{L}}$.

6. An improved estimated set of mixing coefficient vectors is obtained from the transformation $\tilde{c}_{\Gamma_{i}}^{\text {est }} \rightarrow\left(1-\xi_{i}\right) \tilde{c}_{\Gamma_{i}}^{\text {new }}+\xi_{i} \tilde{c}_{\Gamma_{i}}^{\text {est }}$, where $0 \leq \xi_{i}<1$ are also damping or accelerating factors.

The Lagrange multipliers $\epsilon_{a a}$ can be interpreted as approximate electron removal energies only in the average level (AL) approximation, when $n_{\mathrm{L}}=n_{c}$. This is defined by the choice

$$
d_{r s} \equiv \begin{cases}\left(2 J_{r}+1\right) / \sum_{t=1}^{n_{c}}\left(2 J_{t}+1\right) & \text { if } r=s \\ 0 & \text { otherwise. }\end{cases}
$$

We are then effectively optimising on the trace of the full Dirac-Coulomb Hamiltonian matrix (with one element for each value of $M$ ) which is invariant under the orthogonal transformation which diagonalises it. Steps 5 and 6 are then redundant.

The absolute difference, $\left|\epsilon_{i}-\epsilon_{j}\right|$ between a pair of Lagrange multipliers can be interpreted as the energy of the photon that is exchanged in the transverse interaction $(2-5)$. Running GRASP ${ }^{2}$ in the $\mathrm{AL}$ mode for $\mathrm{Ar}^{16+}$, and diagonalizing the matrix $\tilde{H}^{\mathrm{DC}}+\tilde{H}^{\text {trans }}$, one obtaius the approximate ASFs and energies

$$
\begin{aligned}
& \left|\left(00000000.00 \mathrm{~cm}^{-1}\right) 10^{+}\right\rangle=0.9999\left|1 \mathrm{~s}_{1 / 2}^{2} 0^{+}\right\rangle-0.0166\left|1 \mathrm{~s}_{1 / 2} 2 \mathrm{~s}_{1 / 2} 0^{+}\right\rangle \\
& \left|\left(25022573.82 \mathrm{~cm}^{-1}\right) 21^{+}\right\rangle=\left|1 \mathrm{~s}_{1 / 2} 2 \mathrm{~s}_{1 / 2} 1^{+}\right\rangle \\
& \left|\left(25175203.93 \mathrm{~cm}^{-1}\right) 20^{-}\right\rangle=\left|1 \mathrm{~s}_{1 / 2} 2 \mathrm{p}_{1 / 2} 0^{-}\right\rangle \\
& \left|\left(25180420.69 \mathrm{~cm}^{-1}\right) 21^{-}\right\rangle=0.8819\left|1 \mathrm{~s}_{1 / 2} 2 \mathrm{p}_{1 / 2} 1^{-}\right\rangle-0.4715\left|1 \mathrm{~s}_{1 / 2} 2 \mathrm{p}_{3 / 2} 1^{-}\right\rangle \\
& \left|\left(25192331.99 \mathrm{~cm}^{-1}\right) 20^{+}\right\rangle=0.9999\left|1 \mathrm{~s}_{1 / 2} 2 \mathrm{~s}_{1 / 2} 0^{+}\right\rangle+0.0166\left|1 \mathrm{~s}_{1 / 2}^{2} 0^{+}\right\rangle, \\
& \left|\left(25202522.72 \mathrm{~cm}^{-1}\right) 22^{-}\right\rangle=\left|1 \mathrm{~s}_{1 / 2} 2 \mathrm{p}_{3 / 2} 2^{-}\right\rangle, \\
& \left|\left(25312695.15 \mathrm{~cm}^{-1}\right) 21^{-}\right\rangle=0.8819\left|1 \mathrm{~s}_{1 / 2} 2 \mathrm{p}_{3 / 2} 1^{-}\right\rangle+0.4715\left|1 \mathrm{~s}_{1 / 2} 2 \mathrm{p}_{1 / 2} 1^{-}\right\rangle .
\end{aligned}
$$

The excited states' energies in (4-21) are all too high (cf. (4-10)) by amounts varying from $\approx 9000-14000 \mathrm{~cm}^{-1}$. This is largely due to the greater sensitivity of the lowest-lying state to correlation effects. Note, however, that the fine-structure splittings between the lowest three states $J^{-}$are incorrect by only $\approx 400 \mathrm{~cm}^{-1}:$ high quality fine-structure splittings are characteristic of AL calculations. The remaining discrepancy is due to physical effects that are not accounted for - as described above in the last paragraph of section 2. For many purposes, however, the results of such $\mathrm{AL}$ calculations are quite acceptable. GRASP ${ }^{2} \mathrm{AL}$ calculations with $n_{\mathrm{c}} \approx 800$ are routinely performed at Oxford.

Consider the problem of improving the estimate of the $\left|10^{+}\right\rangle$state in $\mathrm{Ar}^{16+}$. As Fischer /23/ has shown, the exact ground-state eigenfunction of a Hamiltonian for a twoelectron atom is obtained in the limit $n_{\max } \rightarrow \infty$ of

$$
\left|10^{+}\right\rangle=\sum_{n=1}^{n_{\max }} \sum_{j=1 / 2}^{n-1 / 2}\left|(n l j)^{2} 0^{+}\right\rangle
$$

The optimal level (OL) method - which allows us to improve the energy of a single level at the cost of obtaining poorer estimates of all other levels included in the calculation - 
Table 1. A sequence of GRASP ${ }^{2}$ OL calculations together with an extrapolation procedure can be used to estimate eigenenergies to high precision: we obtain $E_{\text {exact }}^{\mathrm{DC}}=-68967940 \pm$ $10 \mathrm{~cm}^{-1}$ and $E_{\text {exact }}^{\mathrm{DC}+\text { Breit }}=-68953000 \pm 100 \mathrm{~cm}^{-1}$.

\begin{tabular}{ccccccccccccc}
\hline \hline$n_{\max }$ & \multicolumn{2}{c}{$E_{n_{\max }}^{\mathrm{DC}}$} & \multicolumn{2}{c}{$E_{\infty}^{\mathrm{DC}}$} & \multicolumn{2}{c}{$E_{n_{\max }}^{\mathrm{DC}+\text { Breit }}$} & \multicolumn{3}{c}{$E_{\infty}^{\text {DC+Breit }}$} \\
\hline 1 & -68 & 957 & 877.711 & -68 & 957 & 877.711 & -68 & 941 & 637.091 & -68 & 941 & 637.091 \\
2 & -68 & 965 & 935.542 & -68 & 967 & 086.661 & -68 & 950 & 377.686 & -68 & 953 & 149.956 \\
3 & -68 & 967 & 260.013 & -68 & 967 & 920.028 & -68 & 951 & 896.372 & -68 & 952 & 722.269 \\
4 & -68 & 967 & 639.013 & -68 & 967 & 953.165 & -68 & 952 & 373.162 & -68 & 952 & 826.318 \\
5 & -68 & 967 & 782.962 & -68 & 967 & 941.925 & -68 & 952 & 579.372 & -68 & 952 & 879.571 \\
6 & -68 & 967 & 847.974 & -68 & 967 & 935.814 & -68 & 952 & 689.461 & -68 & 952 & 929.859 \\
\hline
\end{tabular}

is obtained by setting $n_{\mathrm{L}}=1$ in Eq. (4-19). This is clearly the method of choice for the problem at hand. We carry out a series of OL calculations with $n_{\max }=1, \ldots, 6$; the results are listed in column 2 of Table 1 . The differences between consecutive pairs of entries are fitted to a polynomial with terms $a_{k} /(n-1 / 2)^{4+k} / 24 /$. Summing the polynomial over all values of $n>n_{\max }$ yields the entries in column 3 of Table 1.

Because it is now impossible to interpret the Lagrange multipliers $\epsilon_{a a}$ as one-electron removal energies, we are constrained to use Eq. (2-6) - which is independent of the energy of the exchanged photon - to compute effects of higher order. In addition, because we are using perturbation methods, it is necessary to go beyond the expansion (4-22) and include all possible CSFs in a systematic manner; the appropriate expansion is

$$
\left|10^{+}\right\rangle=\sum_{n_{1}, n_{2}=1}^{n_{\max }} \sum_{j=1 / 2}^{\min \left(n_{1}, n_{2}\right)-1 / 2}\left|\left(n_{1} l j\right)\left(n_{2} l j\right) 0^{+}\right\rangle
$$

In column 4 of Table 1 we exhibit the results of the corresponding sequence of computations. The terms of the fitting polynomial are now of the form $a_{k} /(n-1)^{3+k}$. Extrapolated results are listed in column 5 .

Extended AL (EAL) results are obtained when the weights $\left(2 J_{r}+1\right)$ in Eq. (4-20) are replaced by $w_{r}$ chosen by the user. Extended OL (EOL) calculations are obtained when $1<n_{\mathrm{L}}<n_{\mathrm{c}}$ in Eq. (4-19).

\section{Acknowledgments}

Charlotte Fischer pointed out the necessity of computing perturbation corrections with the complete expansion of Eq. (4-23). We are grateful to Claude Zeippen for his translation of the Abstract into French. FAP would like to record his thanks to the organisers of the Third Atomic Workshop and the support staff at Meudon for their hospitality and their efforts in arranging a successful conference. FAP's travel and living expenses were borne by the United Kingdom Science and Engineering Research Council (SERC). FAP is supported 
by a postdoctoral research assistantship from the SERC. All computation has been carried out on the Oxford University Computing Service VAX and CONVEX machines.

\section{References}

/1/ GRANT, I.P., and QUINEY, H.M., Adv. At. Mol. Phys. 23 (1988) 37.

/2/ DIRAC, P.A.M., Proc. Roy. Soc. $\underline{A 117}$ (1928) 610.

/3/ BERESTETSKII, V.B., LIFSHITZ, E.M., and PITAEVSKII, L.P., Relativistic Quantum Theory, Pergamon, Oxford, 1971.

/4/ BREIT, G., Phys. Rev. $\underline{34}$ (1929) 553; ibid. $\underline{36}$ (1930) 383; ibid. $\underline{39}$ (1932) 616.

/5/ GAUNT, J.A., Proc. Roy. Soc. (London) A122 (1929) 513.

/6/ GRANT, I.P., pp. 1-71 in Methods in Computational Chemistry, Vol. 2, (ed. S. Wilson), Plenum, New York, 1988.

/7/ GRANT, I.P., Comput. Phys. Commun. 4 (1972) 377. Erratum: ibid. 14 (1978) 311.

18/ GRANT, I.P., Comput. Phys. Commun. $\underline{5}$ (1973) 263. Erratum: ibid. $\underline{13}$ (1978) 429.

/9/ GRANT, I.P., Comput. Phys. Commun. 11 (1976) 397. Erratum: ibid. 14 (1978) 312.

/10/ PYPER, N.C., GRANT, I.P., and BEATHAM N., Comput. Phys. Commun. 15 (1978) 387.

/11/ CHANG, J.J., Comput. Phys. Commun. 7 (1974) 225.

/12/ McKENZIE, B.J., GRANT, I.P., and NORRINGTON, P.H., Comput. Phys. Commun. 21 (1980) 233.

/13/ BEATHAM, N., GRANT, I.P., McKENZIE, B.J., and PYPER, N.C., Comput. Phys. Commun. 18 (1979) 245.

/14/ GRANT, I.P., and McKENZIE, B.J., J. Phys. B: At. Mol. Phys. 13 (1980) 2671.

/15/ GRANT, I.P., McKENZIE, B.J., NORRINGTON, P.H., MAYERS, D.F., and PYPER, N.C., Comput. Phys. Commun. 21 (1980) 207.

/16/ DYALL, K.G., GRANT, I.P., JOHNSON, C.T., PARPIA, F.A., and PLUMMER, E.P., Comput. Phys. Commun. $\underline{55}$ (1989) 425.

17/ DYALL, K.G., Comput. Phys. Commun. 39 (1986) 141.

/18/ BAR-SHALOM, A., and KLAPISCH, M., Comput. Phys. Commun. 50 (1988) 375.

/19/ BURKE, P.G., Comput. Phys. Commun. 1 (1970) 241. Erratum: ibid. 2 (1971) 173.

/20/ FISCHER, C.F., Comput. Phys. Rep. 3 (1986) 273.

/21/ RAMANA, M.V., RAJAGOPAL, A.K., Adv. Chem. Phys. $\underline{54}$ (1983) 231.

/22/ DRAKE, G.W.F., Can. J. Phys. 66 (1988) 586.

/23/ FISCHER, C.F., The Hartree-Fock Method for Atoms, Wiley, New York, 1977.

/24/ PARPIA, F.A., and GRANT, I.P., J. Phys. B: At. Mol. Opt. Phys. 23 (1990) 211. 